\title{
1 Microbial control over host diet selection
}

2

3 Brian K. Trevelline ${ }^{\#}$ and Kevin D. Kohl

4

5 Department of Biological Sciences, University of Pittsburgh, Pittsburgh, Pennsylvania,

6 15260, USA

7

$8 \quad$ \#Address correspondence to Brian K. Trevelline, brian.trevelline@gmail.com.

9

10

11

12

13

14

15

16

17

18

19

20

21

22

23 


\section{Abstract}

25 Diet selection is a fundamental aspect of animal behavior with numerous

26 ecological and evolutionary implications ${ }^{1}$. While the underlying mechanisms are

27 complex, the availability of essential dietary nutrients can strongly influence diet

28 selection $^{2}$. The gut microbiome has been shown to metabolize many of these

29 same nutrients ${ }^{3}$, leading to the untested hypothesis that intestinal microbiota may

30 influence diet selection ${ }^{4,5}$. Here we show that germ-free mice colonized by gut

31 microbiota from three rodent species with distinct foraging strategies

32 differentially selected diets that varied in macronutrient composition. Specifically,

33 we found that herbivore-conventionalized mice voluntarily selected a higher

34 protein:carbohydrate ratio diet, while omnivore- and carnivore-conventionalized

35 mice selected a lower P:C ratio diet. In support of the long-standing hypothesis

36 that tryptophan - the essential amino acid precursor of serotonin - serves as a

37 peripheral signal regulating diet selection ${ }^{6}$, bacterial genes involved in tryptophan

38 metabolism and plasma tryptophan availability prior to the selection trial were

39 significantly correlated with subsequent voluntary carbohydrate intake. Finally,

40 herbivore-conventionalized mice exhibited larger intestinal compartments

41 associated with microbial fermentation, broadly reflecting the intestinal

42 morphology of their donor species. Together, these results demonstrate that gut

43 microbiome can influence host diet selection and intestinal physiology by

44 mediating the availability of essential amino acids, thereby revealing a novel

45 mechanism by which the gut microbiota influence host foraging behavior. 
Proper nutrition is essential to life, and thus animals have evolved complex

48 internal sensory systems that help maintain nutritional homeostasis by regulating

49 macronutrient intake ${ }^{7}$. The intestinal tract plays a critical role in this process by liberating

50 dietary nutrients (e.g., essential amino acids) that communicate the quality of a meal to

51 central nervous system by direct stimulation of enteric nerves ${ }^{8}$ or through post-

52 absorptive peripheral signals ${ }^{9}$. The intestinal tract also harbors trillions of

53 microorganisms (collectively known as the gut microbiome), which have been shown to

54 influence numerous aspects of host behavior, most likely through metabolites that

55 interact with host sensory systems ${ }^{3}$. Given the importance of dietary nutrients in the

56 regulation of food intake and diet selection ${ }^{2}$, the gut microbiome may influence host

57 foraging behavior through metabolic processes that affect the availability of nutrients

58 recognized by the central nervous system ${ }^{4,5}$. It has been suggested that gut microbiota

59 could employ this strategy to elicit host foraging behaviors that enrich the intestinal

60 environment in nutrients on which they depend (i.e., promoting their own fitness) ${ }^{4}$, while

61 others have posited that a positive-feedback relationship between dietary nutrients and

62 microbial community composition eventually results in stable microbial communities and

63 host foraging behaviors ${ }^{5}$. Regardless, the notion that microbiota may affect host

64 foraging has enormous implications for our understanding of the ecological and

65 evolutionary processes shaping animal behavior, and possibly human health. However,

66 these potential mechanisms operate under the assumption that the gut microbiome

67 influences diet selection - a hypothesis that has existed for years ${ }^{4,5}$, but has never been

68 tested. 
The transplantation of microbiota into germ-free mice is the best available

70 approach for disentangling the effects of the gut microbiome on host behavioral

71 phenotypes from other potentially confounding factors (e.g., host genetics) ${ }^{10}$. To

72 determine whether the gut microbiome influences diet selection, we chose three rodent

73 species with distinct foraging strategies as microbial donors for germ-free mice: a

74 carnivore/insectivore (southern grasshopper mouse, Onychomys torridus), an omnivore

75 (white-footed mouse, Peromyscus leucopus), and an herbivore (montane vole, Microtus

76 montanus). These three species are in the same taxonomic family (Cricetidae) and are

77 all equally distantly related to lab mice (Mus musculus, family Muridae $)^{11}$. Under sterile

78 laboratory conditions, we randomly divided 30 adult male germ-free mice into Carn-

79 CONV, Omni-CONV, and Herb-CONV treatment groups ( $\mathrm{n}=10$ mice per group), where

80 each mouse in a given group was "conventionalized" (i.e., inoculated) with the cecal

81 contents of a unique, wild-caught donor individual (to better reflect natural interindividual

82 variation) (Fig. 1a). Conventionalized mice were acclimated to their microbiota for 7

83 days, during which they were offered only sterile water and a low protein:carbohydrate

84 ratio diet (LPC; Extended Data Table 1). There were no differences in daily or

85 cumulative macronutrient intake across treatment groups during the acclimation period

86 (Extended Data Fig. 1; Supplemental Data 1). After acclimation, conventionalized mice

87 were given a choice between the LPC diet and one with a higher P:C ratio (HPC;

88 Extended Data Table 1) for a period of 11 days (Fig. 1a).

89 To determine whether treatment groups differed in foraging behavior, we

90 employed a state-space approach known as the Geometric Framework for nutrition ${ }^{12}$,

91 which allowed us to measure the effect of the microbiome on carbohydrate and protein 
92 intake. Supporting the hypothesis that the gut microbiome influences diet selection, this

93 approach revealed statistically significant differences in macronutrient intake across

94 groups of conventionalized mice (Fig. 1b). Treatment groups differed significantly in

95 daily (Extended Data Fig. 1) and cumulative carbohydrate intake (Fig. 1b) during the

96 diet selection trial, with Herb-CONV mice consuming fewer carbohydrates than the

97 other groups (Fig. 1b inset). Treatment groups did not differ in either daily (Extended

98 Data Fig. 1) or cumulative protein intake (Fig. 1b inset). Lower cumulative carbohydrate

99 intake among Herb-CONV mice was driven by their selection of a higher P:C ratio diet

100 compared to Omni-CONV and Carn-CONV mice (Fig. 1b). The preference of Herb-

101 CONV mice for the HPC diet are consistent with previous studies showing that Microtus

102 voles prefer high-protein foods when available ${ }^{13,14}$, and the opportunistic consumption of

103 high-protein meals among wild herbivores ${ }^{15,16}$.

104 Next, we characterized day 0 gut microbial community structure, microbiome

105 function, and plasma metabolites of conventionalized mice to determine how these

106 aspects were associated with differential diet selection across treatment groups. 16S

107 rRNA inventories confirmed that both donors and recipients harbored distinct bacterial

108 communities, with recipients most closely resembling the communities of their donor

109 species (Fig. 1c, Extended Data Fig. 2, Extended Data Table 2). Bacterial ASV richness

110 and phylogenetic diversity were similar between Carn-CONV and Omni-CONV mice,

111 but significantly lower in Herb-CONV mice (Extended Data Fig. 3). The bacterial

112 communities of conventionalized mice were dominated by the phyla Bacteroidetes and

113 Firmicutes (Extended Fig. 4a), with differences in the microbiome community structure

114 across treatment groups being driven by 13 bacterial families in the phyla 
115 Bacteroidetes, Firmicutes, and Actinobacteria (Extended Data Fig. 4b, c). Metagenomic

116 analysis of recipient fecal samples revealed a statistically significant effect of donor

117 species on the relative abundances of 176 (49\%) KEGG functional modules (Fig. 1d;

118 Supplemental Data 2). These differences in microbiome community structure and

119 function were accompanied by concomitant differences in plasma metabolites (Fig. 1e),

120 with 24 identified metabolites (14\%) differing significantly across treatment groups

121 (Supplemental Data 3). Together, these results demonstrate that interspecific

122 differences in gut microbial communities across rodents with divergent foraging

123 strategies translate to distinct microbial functions and metabolite profiles independent of

124 host diet.

125 There is substantial evidence that the availability of circulating essential amino

126 acids (EAAs) provide peripheral signals that act to regulate macronutrient intake and

127 diet selection ${ }^{9}$. Despite consuming identical diets prior to the selection trial, treatment

128 groups differed in circulating levels of several amino acids, with Herb-CONV mice

129 exhibiting significantly higher amounts of the EAAs isoleucine and tryptophan (Fig. 2a),

130 though tryptophan was only significant after the removal of two exceptionally-low

131 statistical outliers $(>1.5 \times$ IQR) from the Herb-CONV group. While EAAs are primarily

132 derived from the diet, bacteria can also produce these peptides through their own

133 metabolic processes ${ }^{17}$, and thus the gut microbiome may act as a source of EAAs for

134 their hosts. In support of this hypothesis, treatment groups exhibited broad differences

135 in the microbial synthesis and degradation of EAAs (Fig. 2b). Notably, the microbiome

136 of Herb-CONV mice had a higher abundance of genes involved in the synthesis of

137 aromatic amino acids (phenylalanine, tryptophan, and tyrosine) (Fig. 2b), all of which 
138 are synthesized from chorismate (product of the Shikimate pathway) ${ }^{18}$. The ratios of

139 bacterial genes involved in tryptophan biosynthesis (M00023) to those involved in

140 tryptophan degradation via the kynurenine pathway (M00038) were significantly

141 correlated with plasma tryptophan (Fig. 2c). Given that conventionalized mice

142 consumed identical diets prior to blood collections, these results demonstrate that

143 bacterial metabolism can alter the availability of circulating levels of plasma EAAs.

144 There is emerging evidence that bacterial tryptophan metabolism is a key

145 mechanism by which the gut microbiome can influence host behavior ${ }^{19,20}$. This

146 relationship is a consequence of tryptophan's role as the primary regulatory molecule

147 for the synthesis of central serotonin (5-hydroxytryptamine, 5-HT) ${ }^{21}$, which has been

148 shown to drive foraging behavior and diet selection in several experimental studies ${ }^{6}$. For

149 example, when given a choice between low- or high-carbohydrate meals, rats receiving

150 hypothalamic injections of 5-HT significantly reduced their carbohydrate intake ${ }^{22}$.

151 Importantly, serotonin synthesis is extraordinarily sensitive to plasma tryptophan

152 availability, and thus plasma tryptophan is generally considered a reliable proxy for

153 central serotonin ${ }^{23}$. Therefore, we predicted that plasma tryptophan would be

154 associated with differences in diet selection among conventionalized mice. Indeed, we

155 found a statistically significant correlation between day 0 plasma tryptophan and 156 subsequent voluntary carbohydrate and P:C intake (Fig. 2c). More recent work has

157 argued that serotonin synthesis is affected by the availability of tryptophan relative to

158 the large neutral amino acids (LNAA: Leu, Ile, Phe, Tyr, and Val) that compete for

159 transport across the blood brain barrier ${ }^{24}$. Consistent with these studies, we found a

160 statistically significant correlation between day 0 Trp:LNAA ratios, cumulative 
161 carbohydrate intake, and P:C intake (Fig. 2c). Further, the ratio of tryptophan

162 biosynthesis and degradation modules were also statistically significant predictors of

163 carbohydrate and $\mathrm{P}: \mathrm{C}$ intake (Fig. 2c). Overall, these results strongly support the

164 hypothesis that bacterial tryptophan metabolism influences host diet selection. Interspecific differences in foraging behavior are generally associated with diet-

166 specific adaptations to intestinal physiology. For example, herbivores generally maintain

167 an enlarged cecum (fermentation chamber) that enhances the digestibility of low-quality,

168 carbohydrate-rich foods ${ }^{25}$. Given that the gut microbiome can profoundly alter host

169 intestinal gene expression and physiology ${ }^{26-28}$, divergent microbial communities may

170 drive differences in intestinal morphology across feeding strategies. At the conclusion of

171 the diet selection trial (day 11), we quantified intestinal morphology with the prediction

172 that conventionalized mice would exhibit differences that broadly reflected that of their

173 donor species. Consistent with evolutionary adaptations to herbivory, treatment groups

174 differed significantly in empty cecum mass (Fig. 3a), empty colon mass (Fig. 3b), and

175 colon length (after the removal of an exceptionally-high statistical outlier from the Carn-

176 CONV group) (Fig. 3c), with comparatively larger intestinal compartments among the

177 Herb-CONV mice. There was no change in body mass over the duration of the

178 experiment $(F=1.66, P=0.327)$. The obvious question is whether the gut microbiome

179 affected intestinal morphology directly or via differential diet selection. While our

180 experimental design makes it difficult to disentangle the effects of differential diet

181 selection from those of microbiome, it is worth noting that previous work has

182 demonstrated that lab mice fed low $P: C$ ratio diets had larger intestinal compartments

183 (e.g., cecum and colon) compared to those fed higher $\mathrm{P}: \mathrm{C} \operatorname{diets}^{28}$. In our study, we 
184 observed the opposite - Herb-CONV mice, which consumed a higher P:C ratio diet (Fig.

185 1b), exhibited larger cecum and colon masses (Fig. 3). These results contradict the

186 generally accepted model of adaptive physiological responses to dietary carbohydrates,

187 suggesting that the gut microbiome may drive interspecific differences in host intestinal 188 physiology independent from those of diet and genetics.

189 The gut microbiome is now recognized as a key player in host digestion, 190 performance, and health ${ }^{29}$, leading to the realization that animals should no longer be

191 thought of as singular entities, but rather as 'holobionts' - complex genetic and

192 biomolecular networks composed of the host plus its associated microbiota, upon which

193 natural selection acts $^{30}$. In support of these concepts, our study provides the first

194 evidence that variation in the gut microbiome can influence diet selection, which is a 195 fundamental aspect of host behavior. Specifically, we showed that host species196 associated gut microbiota can produce statistically significant differences in the diet 197 selection of conventionalized mice in just 11 days, likely through differential bacterial 198 metabolism and downstream availability of EAAs, especially tryptophan. These findings

199 provide evidence of a mechanistic link between the gut microbiome and animal 200 behavior, with implications for large-scale evolutionary processes involving diet, 201 nutrition, and foraging ecology. 


\section{REFERENCES}

2111 Stephens, D. W., Brown, J. S. \& Ydenberg, R. C. Foraging: behavior and

212 ecology. (University of Chicago Press, 2008).

2132 Berthoud, H. R., Münzberg, H., Richards, B. K. \& Morrison, C. D. Neural and

214 metabolic regulation of macronutrient intake and selection. Proceedings of the

$215 \quad$ Nutrition Society 71, 390-400 (2012).

2163 Cryan, J. F. et al. The microbiota-gut-brain axis. Physiological Reviews 99, 18772013 (2019).

2184 Alcock, J., Maley, C. C. \& Aktipis, C. A. Is eating behavior manipulated by the 219 gastrointestinal microbiota? Evolutionary pressures and potential mechanisms.

220 BioEssays 36, 940-949 (2014).

2215 Norris, V., Molina, F. \& Gewirtz, A. T. Hypothesis: bacteria control host appetites. 222 Journal of Bacteriology 195, 411-416 (2013).

2236 Blundell, J. E. \& Halford, J. C. Serotonin and appetite regulation. CNS Drugs 9, $224 \quad 473-495(1998)$.

2257 Kyriazakis, I., Tolkamp, B. J. \& Emmans, G. Diet selection and animal state: an 226 integrative framework. Proceedings of the Nutrition Society 58, 765-772 (1999).

2278 Furness, J. B. The enteric nervous system and neurogastroenterology. Nature 228 Reviews Gastroenterology \& Hepatology 9, 286 (2012). 
2299 Stubbs, R. J. Peripheral signals affecting food intake. Nutrition 15, 614-625

$230 \quad$ (1999).

23110 Luczynski, P. et al. Growing up in a bubble: using germ-free animals to assess

232 the influence of the gut microbiota on brain and behavior. International Journal of

$233 \quad$ Neuropsychopharmacology 19 (2016).

23411 Steppan, S. J., Adkins, R. M. \& Anderson, J. Phylogeny and divergence-date

235 estimates of rapid radiations in muroid rodents based on multiple nuclear genes.

$236 \quad$ Systematic Biology 53, 533-553 (2004).

23712 Simpson, S. J. \& Raubenheimer, D. A multi-level analysis of feeding behaviour:

238 the geometry of nutritional decisions. Philosophical Transactions of the Royal

239 Society of London. Series B: Biological Sciences 342, 381-402 (1993).

24013 Harju, A. \& Hakkarainen, O. Effect of protein and birch-bark powder on selection

241 of food by root voles (Microtus oeconomus). Journal of Mammalogy 78, 563-568

$242 \quad$ (1997).

24314 Bergeron, J. \& Jodoin, L. Defining "high quality" food resources of herbivores: the

244 case for meadow voles (Microtus pennsylvanicus). Oecologia 71, 510-517

$245 \quad$ (1987).

$24615 \mathrm{Nie}$, Y. et al. Giant pandas are macronutritional carnivores. Current Biology 29,

$247 \quad$ 1677-1682. e1672 (2019).

24816 Ulappa, A. C. et al. Plant protein and secondary metabolites influence diet 249 selection in a mammalian specialist herbivore. Journal of Mammalogy 95, 834-

$250 \quad 842(2014)$. 
25117 Dai, Z. L., Wu, G. \& Zhu, W. Y. Amino acid metabolism in intestinal bacteria: links

252 between gut ecology and host health. Frontiers in Bioscience 16, 1768-1786

$253 \quad$ (2011).

25418 Herrmann, K. M. \& Weaver, L. M. The shikimate pathway. Annual Review of

$255 \quad$ Plant Biology 50, 473-503 (1999).

25619 Gao, K., Mu, C. L., Farzi, A. \& Zhu, W. Y. Tryptophan metabolism: a link between

257 the gut microbiota and brain. Advances in Nutrition (2019).

25820 O'Mahony, S. M., Clarke, G., Borre, Y., Dinan, T. \& Cryan, J. Serotonin,

259 tryptophan metabolism and the brain-gut-microbiome axis. Behavioural Brain

$260 \quad$ Research 277, 32-48 (2015).

26121 Fernstrom, J. D. Role of precursor availability in control of monoamine

262 biosynthesis in brain. Physiological Reviews 63, 484-546 (1983).

26322 Shor-Posner, G., Grinker, J. A., Marinescu, C., Brown, O. \& Leibowitz, S. F.

$264 \quad$ Hypothalamic serotonin in the control of meal patterns and macronutrient

265 selection. Brain Research Bulletin 17, 663-671 (1986).

26623 Fernstrom, J. D. \& Wurtman, R. Brain serotonin content: physiological

267 dependence on plasma tryptophan levels. Science 173, 149-152 (1971).

26824 Fernstrom, J. D. Large neutral amino acids: dietary effects on brain

269 neurochemistry and function. Amino Acids 45, 419-430 (2013).

27025 Stevens, C. E. \& Hume, I. D. Comparative physiology of the vertebrate digestive

$271 \quad$ system. (Cambridge University Press, 2004).

27226 Chevalier, C. et al. Gut microbiota orchestrates energy homeostasis during cold.

$273 \quad$ Cell 163, 1360-1374 (2015). 
27427 Broderick, N. A., Buchon, N. \& Lemaitre, B. Microbiota-induced changes in

275 Drosophila melanogaster host gene expression and gut morphology. MBio 5,

276 e01117-01114 (2014).

27728 Sørensen, A., Mayntz, D., Simpson, S. J. \& Raubenheimer, D. Dietary ratio of 278 protein to carbohydrate induces plastic responses in the gastrointestinal tract of 279 mice. Journal of Comparative Physiology B 180, 259-266 (2010).

28029 McFall-Ngai, M. et al. Animals in a bacterial world, a new imperative for the life

281 sciences. Proceedings of the National Academy of Sciences 110, 3229-3236 $282 \quad$ (2013).

28330 Bordenstein, S. R. \& Theis, K. R. Host biology in light of the microbiome: ten 284 principles of holobionts and hologenomes. PLoS Biology 13, e1002226 (2015). 

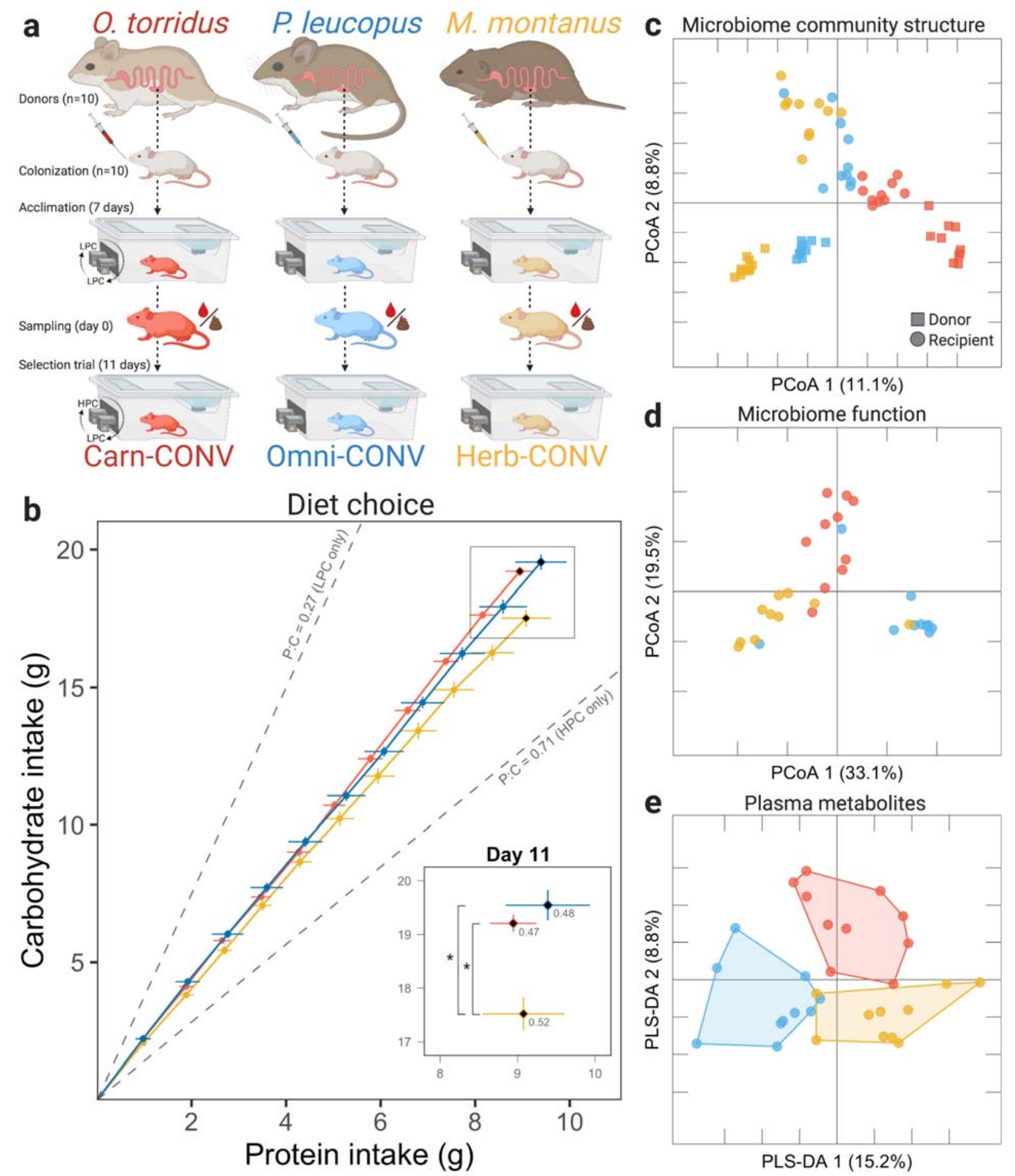

Fig. 1. The gut microbiome influences host diet selection. a, Overview of 
290 species of wild rodents with distinct foraging strategies: carnivorous Onychomys torridus

291 (Carn-CONV), omnivorous Peromyscus leucopus (Omni-CONV), and herbivorous

292 Microtus montanus (Herb-CONV). Conventionalized mice were acclimated on LPC diet

293 for 7 days before day 0 blood and fecal sampling. Conventionalized mice were then

294 given a choice between LPC and HPC diets for 11 days. Daily diet intakes were tracked

295 via two feeder hoods, which were rotated daily to avoid learned preferences. b,

296 Treatment groups differed significantly in macronutrient intake (Wilks' $\lambda=0.67, \mathrm{P}=$

297 0.037), with Herb-CONV mice voluntarily consuming fewer carbohydrates than the

298 Omni- and Carn-CONV groups $(F=5.42, P=0.011)$. There was no difference in

299 cumulative protein intake across treatment groups $(F=0.72, P=0.497)$. Dashed rails

300 and associated $\mathrm{P}: \mathrm{C}$ ratios indicate the expected result if mice consumed only a single

301 diet, while $\mathrm{P}: \mathrm{C}$ ratios next to the cumulative macronutrient intake points for each

302 treatment group indicates observed P:C ratio intake. c, Principal coordinate analysis

303 (PCoA) of 16S rRNA inventories of wild donors (squares) and conventionalized

304 recipients at day 0 (circles) using Bray-Curtis dissimilarity. Microbial community

305 structure differed significantly among wild donors (Pseudo-F $=8.45, P=0.001$ ) and

306 recipients (Pseudo-F $=3.55, P=0.001$ ), with recipients most closely resembling the

307 communities of their donor species (Extended Data Table 2). d, PCoA analysis showing

308 a statistically significant difference in the relative abundances of microbial KEGG

309 modules using Bray-Curtis dissimilarity (Pseudo-F = 6.48, $P=0.001$ ). e, PLS-DA

310 analysis illustrating broad differences in identified plasma metabolites across

311 conventionalized mice at day $0\left(R^{2}=0.92, Q^{2}=0.30\right)$. * denotes $P \leq 0.05$. 
a

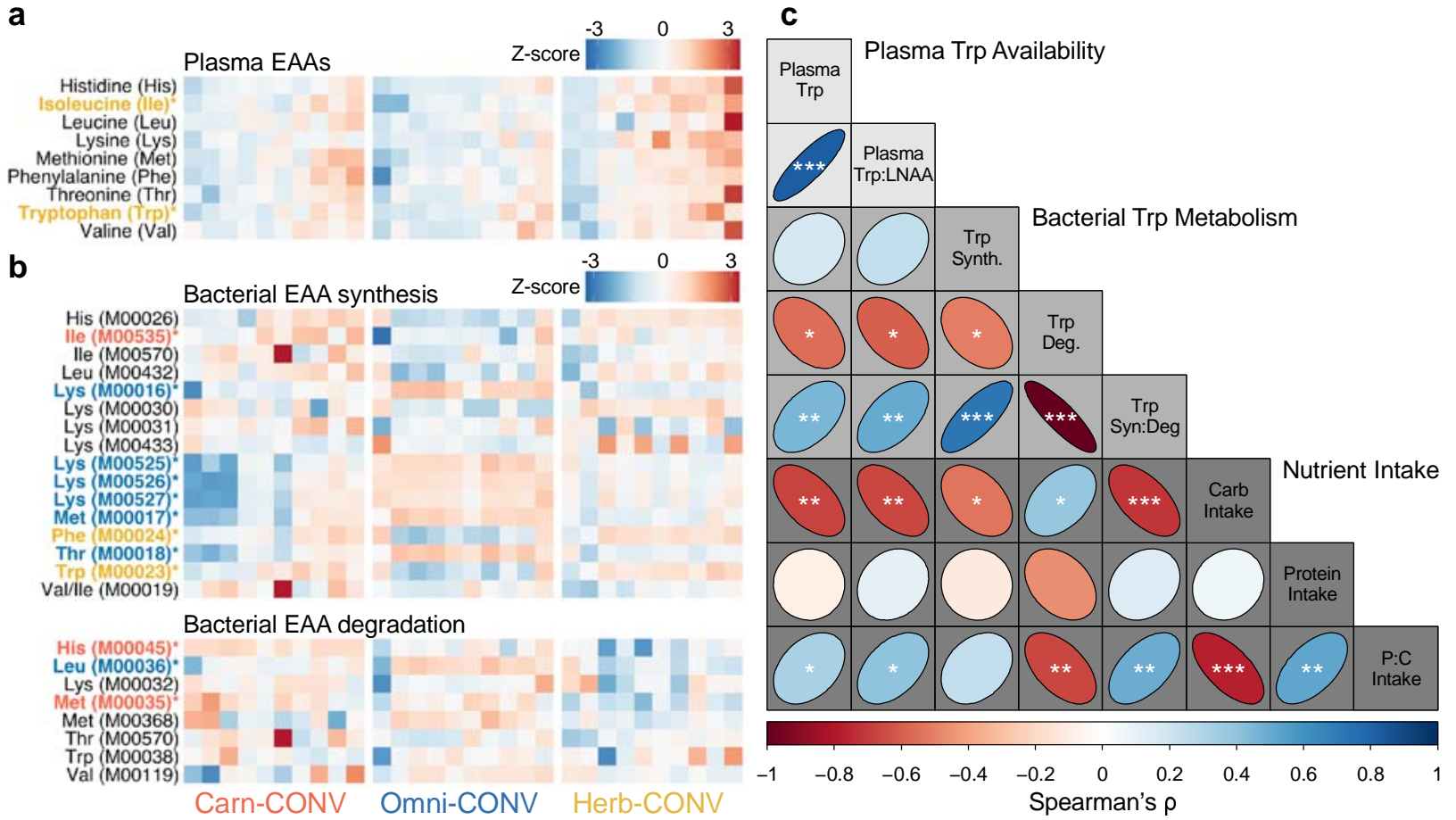

314 Fig. 2. Day 0 plasma tryptophan availability and bacterial tryptophan metabolism

315 are associated with differential macronutrient intake across treatment groups. a,

316 Heatmap illustrating broad differences in plasma levels of essential amino acids across

317 treatment groups, with Herb-CONV mice exhibiting significantly greater levels of

318 isoleucine $\left(X^{2}=7.95, P=0.019\right)$ and tryptophan $\left(X^{2}=7.12, P=0.029\right)$. Columns

319 represent individual conventionalized mice for each treatment group. * denotes $\mathrm{P} \leq 0.05$

320 and color indicates the treatment group with greatest circulating plasma levels $(\mathrm{red}=$

321 Carn-CONV, blue $=$ Omni-CONV, and yellow $=$ Herb-CONV). b, Heatmap illustrating

322 broad differences in the abundances of microbial genes associated with metabolism of

323 essential amino acids (Supplemental Data 3). * denotes $\mathrm{P} \leq 0.05$ and color indicates the

324 treatment group with greatest relative abundance. c, Correlation plot summarizing

325 relationships between plasma tryptophan availability, bacterial tryptophan metabolism,

326 and host diet selection among conventionalized mice. The direction and color of the 
327 ellipses indicate whether correlations were positive or negative, and asterisks indicate

328 whether Spearman's correlations were statistically significant $\left({ }^{*}\right.$ denotes $P \leq 0.05$, **

329 denotes $\mathrm{P}<0.01$, and ${ }^{* * *}$ denotes $\left.\mathrm{P}<0.001\right)$.

330

331

332

333

334

335

336

337

338

339

340

341

342

343

344

345 

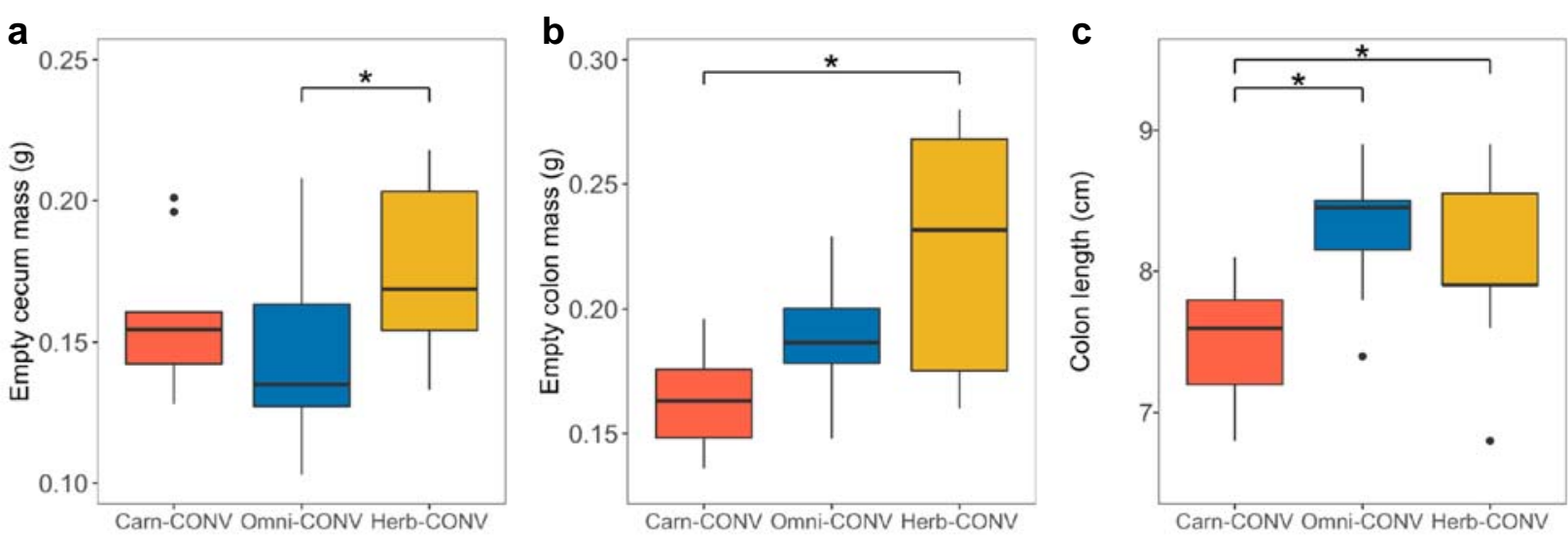

347 Fig. 3. Treatment groups exhibit differences in intestinal morphology that broadly

348 reflect that of their donor species. a, Empty cecum mass differed significantly across

349 treatment groups $(F=4.19, P=0.027)$, with Herb-CONV mice exhibiting a greater

350 cecum mass than Omni-CONV mice. $\mathbf{b}$, Empty colon mass differed significantly across

351 treatment groups $(F=6.65, P=0.005$; Fig. $3 b)$, with Herb-CONV mice exhibiting a

352 greater colon mass than Carn-CONV mice. c, Colon length differed significantly across

353 treatment groups $(F=7.30, P=0.003)$, with Carn-CONV mice exhibiting shorter colons

354 compared to Omni- and Herb-CONV mice. * denotes $\mathrm{P} \leq 0.05$. 
METHODS

Wild rodents. Wild Onychomys torridus were collected in August 2018 from field sites in near Green Valley, Pima Co., AZ (31.802834, -110.891172), Peromyscus

367 leucopus in May 2018 near Murray, Calloway Co., KY (36.686582, -88.221204), and

368 Microtus montanus in July 2018 at Timpie Springs Waterfowl Management Area, 369 Dugway, Tooele Co., UT $(40.753708,-112.639903)$. Ten individuals from each species 370 were collected using baited Sherman live traps under the following state permits: $O$.

371 torridus (AZ Game and Fish Dept., SP627958), P. leucopus (KY Dept. of Fish and

372 Wildlife, SC1911097), and M. montanus (UT Division of Wildlife Resources,

373 1COLL5194-2). Animals were euthanized within 12 hours and immediately dissected

374 under IACUC protocols registered at the University of Utah (16-02011 to D. Dearing),

375 Murray State University (2018-026 to T. Derting), and University of Alabama (18-04-

3761159 to S. Secor). Cecum contents for microbiome transplants were transferred using to

$3771.7 \mathrm{~mL}$ Eppendorf tube using sterile instruments and immediately frozen at $-20^{\circ} \mathrm{C}$.

378 Microbiome transplants. Donor cecum contents were diluted at $100 \mathrm{mg} / \mathrm{mL}$ in 379 sterile phosphate-buffered saline containing $0.2 \mathrm{~g} / \mathrm{L} \mathrm{Na} \mathrm{N}_{2} \mathrm{~S}$ and $0.5 \mathrm{~g} / \mathrm{L}$ cysteine as 380 reducing agents ${ }^{31,32}$. Under sterile laboratory conditions, 30 adult (aged 6-8 weeks) 381 male germ-free C57BL/6 mice (Taconic Biosciences, Inc., Rensselaer, NY) were 382 randomly divided into Carn-CONV, Omni-CONV, and Herb-CONV groups where each

383 mouse in a given group was colonized by oral gavage of $200 \mu \mathrm{L}$ of fecal slurry from a 384 unique, wild-caught donor individual. Conventionalized mice were then singly-housed in 385 sterile static cages (Innovive, Inc., San Diego, CA; MSX2-AD) modified by the addition 386 of two metabolic feeder hoods (Laboratory Products, Inc., Seaford, DE; 2110S) that 
387 prevent mice from caching powdered diets, and thus enable the tracking daily 388 macronutrient intake (see below).

389 Diet selection experiment. After colonization, conventionalized mice were

390 acclimated for 7 days (to allow the gut microbiome to stabilize ${ }^{32}$ ), during which they

391 were offered only sterile water and a low protein:carbohydrate ratio diet (LPC [0.27];

392 Extended Data Table 1). After acclimation (day 0), mice were briefly removed from their

393 cages for a $200 \mu \mathrm{L}$ blood draw for metabolomics analysis (see details below). Mice were

394 weighed (rounded to nearest hundredth) and returned to empty cages to facilitate the

395 collection of fresh fecal samples for 16S rRNA microbial inventories and shotgun

396 metagenomics (see details below). Conventionalized mice were then presented with a

397 choice between two isocaloric diets (Extended Data Table 1): (1) the LPC (0.27) diet

398 offered during acclimation and (2) a diet with a higher $\mathrm{P}: \mathrm{C}$ ratio (HPC [0.71]). The

399 positions of these two diets were rotated daily to avoid learned preferences. Diets were

400 designed by Teklad/Envigo (Indianapolis, IN), and were powdered prior sterilization to

401 be visually indistinguishable from each other and to prevent food caching. Daily food

402 consumption was calculated as the difference between the mass (rounded to nearest

403 thousandth) of each diet presented ( 8 $\mathrm{g})$ and the mass of each diet remaining after a

404 24-hour period. After tracking diet preferences for 11 consecutive days, animals were

405 euthanized and dissected to investigate differences in the empty masses (rounded to

406 nearest thousandth) of intestinal compartments. Conventionalized mice were

407 maintained on a $12: 12-\mathrm{h}$ light:dark cycle, with $21^{\circ} \mathrm{C}$ ambient temperature and $40 \%$

408 humidity for the duration of the experiment. Animal experiments were conducted at the 
409 University of Pittsburgh Plum Borough Primate Facility under IACUC protocol

41019074445.

$411 \quad$ Metabolomics. Blood plasma was analyzed for primary metabolites (amino

412 acids, hydroxyl acids, carbohydrates, sugar acids, sterols, aromatics, nucleosides,

413 amines, and miscellaneous compounds) by the West Coast Metabolomics Center at the

414 University of California - Davis, which performed all sample preparation, data

415 acquisition, and data processing as previously described ${ }^{33}$. Briefly, metabolites were

416 extracted using a mixture of acetonitrile:isopropanol:water $(3: 3: 2, \mathrm{v} / \mathrm{v} / \mathrm{v})$ as well as $1: 1$

417 acetonitrile:water for removal of protein from serum. Dried metabolite extracts were

418 resuspended in methoxyamine hydrochloride in pyridine for derivatization before being

419 analyzed using gas chromatography-time-of-flight (GC-TOF) using a LECO Pegasus IV

420 mass spectrometer equipped with automated liner exchange (ALEX; Gerstel

421 corporation) and cold injection system (CIS; Gerstel corporation) for data acquisition.

422 The CIS temperature was set at $50 \square^{\circ} \mathrm{C}$ to $250 \square^{\circ} \mathrm{C}$ final temperature at a rate of $12 \square^{\circ} \mathrm{C}$

423 s-1. Raw GC-TOF MS data were preprocessed with ChromaTOF (version 2.32) and

424 apex masses were used to identify metabolites using the BinBase database. Values

425 were reported as peak height for the quantification ion ( $\mathrm{m} / \mathrm{z}$ value) at the specific

426 retention index, which is more precise than peak area for low abundant metabolites. All

427 database entries that were positively detected in more than $10 \%$ of the samples of a

428 study design class for unidentified metabolites were reported. Raw peak heights were

429 vector normalized to reduce the impact of between-series drifts of instrument sensitivity,

430 caused by machine maintenance status and tuning parameters. 
DNA extractions. DNA was extracted from donor cecal contents and day 0

432 conventionalized mouse feces using the Qiagen PowerFecal DNA Kit (Qiagen, Hilden,

433 Germany; 12830) following the manufacturer's instructions.

434 16S rRNA microbial inventories. Extracted DNA from conventionalized mice

435 and donor cecum contents was amplified and sequenced by the Genome Research

436 Core of the University of Illinois at Chicago as previously described ${ }^{34}$. Briefly,

437 polymerase chain reaction (PCR) was used to amplify a portion of the bacterial 16S

438 rRNA gene for Illumina sequencing using the Earth Microbiome Project primers 515F

439 (GTGCCAGCMGCCGCGGTAA) and 806R (GGACTACNVGGGTWTCTAAT) targeting

440 the V4 region of microbial small subunit ribosomal RNA gene ${ }^{35}$. Amplicon libraries were

441 sequenced using a 2x251 paired-end run on an Illumina MiSeq. Raw Illumina

442 sequencing reads $(1,977,423)$ were paired and quality filtered via the DADA2 pipeline ${ }^{36}$

443 in QIIME2 (version 2020.2) ${ }^{37}$ using default parameters. Sequences that passed the

444 quality filter were clustered into amplicon sequence variants (ASVs), which were

445 identified using the SILVA reference database (release 132$)^{38}$. Identified ASVs were

446 filtered to exclude non-bacterial sequences (archaea, chloroplast, eukaryote, and

447 mitochondria), reducing our total number of reads to $1,423,306$ (mean of 23,722 per

448 sample $\pm 7,560$ SD) and 4,282 ASVs. We rarefied ASV tables to the sample with fewest

449 number of reads $(4,174)$ before comparisons of alpha (ASV richness and Faith's

450 phylogenetic diversity) and beta diversity (Bray-Curtis and unweighted/weighted $451 \quad$ UniFrac distances $\left.{ }^{39}\right)$.

452 Shotgun metagenomics. Extracted DNA from conventionalized mice was sent 453 to CoreBiome, Inc. (St. Paul, MN) for shotgun metagenomic analysis using 
454 BoosterShot ${ }^{\mathrm{TM}} \square$. Briefly, sequencing libraries were prepared using a procedure adapted

455 from the Illumina Nextera Library Prep Kit (Illumina, 20018705) and sequenced on an

456 Illumina NovaSeq using single-end $1 \times 100$ reads with the Illumina NovaSeq SP reagent

457 kit (Illumina, 20027464). Raw sequences $(125,629,570)$ were filtered for low quality (Q-

458 Score $<30)$ and length $(<50)$, trimmed of adapter sequences, and converted into a

459 single fasta using shi7 (version 0.99$)^{40}$. Sequences were then trimmed to a maximum

460 length of $100 \mathrm{bp}$ and aligned using BURST (version 0.99 .8$)^{41}$ at $97 \%$ identity against

461 CoreBiome's Venti database consisting of all RefSeq bacterial genomes with additional

462 manually curated strains as well as a bacterial $\mathrm{KEGG}^{42}$ annotated database created

463 from de-replicating the bacterial genes within the Venti database. KEGG orthology

464 counts were converted to relative abundance within a sample and collapsed into KEGG

465 modules for statistical analysis.

466 Statistics. Differences in macronutrient intake across treatment groups were 467 tested using a repeated-measures multivariate analysis of variance (MANOVA) while

468 controlling for the effects of body mass and donor species. Microbial community 469 structure (from 16S rRNA inventories) was visualized using principal coordinates 470 analysis (PCoA) on ASV relative abundances, which were then assessed for differences

471 (controlling for multiple comparisons using false discovery rate corrected P-values)

472 across treatment groups using non-parametric permutational multivariate analysis of

473 variance (PERMANOVA), analysis of similarity (ANOSIM), and permutational analysis

474 of dispersion (PERMDISP) in QIIME2 ${ }^{37}$. Microbiome function (from metagenomics) was

475 visualized using PCoA on KEGG module relative abundances and analyzed for

476 differences across treatment groups with PERMANOVA in QIIME2. Differences in the 
477 relative abundance of bacterial phyla and genera, and functional KEGG modules across

478 conventionalized mice were tested using the non-parametric Krustal-Wallis test and

479 linear discriminant analysis in LEfSe using the "one-against-all" strategy for multi-class

480 analysis $^{43}$. Identified plasma metabolites were filtered (based on mean intensity and

481 (QR) and auto-scaled before using non-parametric ANOVAs to identify metabolites that

482 varied significantly across treatment groups and visualized using supervised partial

483 least square discriminant analysis (PLS-DA) in MetaboAnalyst (version 4.0) ${ }^{44}$. Non-

484 parametric Spearman rank correlations between plasma Trp availability, Trp KEGG

485 modules, and macronutrient intake were conducted using non-parametric Spearman's

486 test (controlling for the effect of donor species) in the R package ppcor (version 1.1) ${ }^{45}$

487 and visualized using corrplot (version 0.85$)^{46}$. Differences in empty cecum/colon

488 masses and colon length across treatment groups were tested using ANOVA with body

489 mass as a covariate and corrected for multiple comparisons using Tukey's HSD. Unless

490 otherwise noted, all statistical tests were two-sided and conducted in JMP Pro version

49114.1 .0 (SAS Institute Inc., Cary, NC). For all statistical analyses, P-values $\leq 0.05$ were

492 defined as 'significant'.

493

\section{METHODS REFERENCES}

49531 Tremaroli, V. et al. Roux-en-Y gastric bypass and vertical banded gastroplasty

496 induce long-term changes on the human gut microbiome contributing to fat mass

497 regulation. Cell Metabolism 22, 228-238 (2015).

49832 Molinaro, A. et al. Host-microbiota interaction induces bi-phasic inflammation

499 and glucose intolerance in mice. Molecular Metabolism 6, 1371-1380 (2017). 
50033 Fiehn, O. et al. Quality control for plant metabolomics: reporting MSI-compliant

$501 \quad$ studies. The Plant Journal 53, 691-704 (2008).

50234 Trevelline, B. K., MacLeod, K. J., Langkilde, T. \& Kohl, K. D. Gestation alters the 503 gut microbiota of an oviparous lizard. FEMS Microbiology Ecology 95, fiz086 (2019).

50535 Caporaso, J. G. et al. Global patterns of 16S rRNA diversity at a depth of millions of sequences per sample. Proceedings of the National Academy of Sciences 108, 4516-4522 (2011).

50836 Callahan, B. J. et al. High-resolution sample inference from Illumina amplicon 509 data. Nature Methods 13, 581-583 (2016).

51037 Bolyen, E. et al. Reproducible, interactive, scalable and extensible microbiome 511 data science using QIIME 2. Nature Biotechnology 37, 852-857 (2019).

51238 Quast, C. et al. The SILVA ribosomal RNA gene database project: improved data processing and web-based tools. Nucleic Acids Research 41, D590-D596 (2012).

51439 Lozupone, C. \& Knight, R. UniFrac: a new phylogenetic method for comparing 515 microbial communities. Applied and Environmental Microbiology 71, 8228-8235 $516 \quad$ (2005).

51740 Al-Ghalith, G. A., Hillmann, B., Ang, K., Shields-Cutler, R. \& Knights, D. SHI7 is a 518 self-learning pipeline for multipurpose short-read DNA quality control. MSystems $519 \quad 3$, e00202-00217 (2018).

$52041 \quad$ Al-Ghalith, G. \& Knights, D. BURST enables optimal exhaustive DNA alignment $521 \quad$ for big data. doi.org/10.5281/zenodo.806850 (2017). 
52242 Kanehisa, M. \& Goto, S. KEGG: Kyoto encyclopedia of genes and genomes.

$523 \quad$ Nucleic Acids Research 28, 27-30 (2000).

52443 Segata, N. et al. Metagenomic biomarker discovery and explanation. Genome

$525 \quad$ Biology 12, R60 (2011).

52644 Chong, J., Wishart, D. S. \& Xia, J. Using metaboanalyst 4.0 for comprehensive 527 and integrative metabolomics data analysis. Current Protocols in Bioinformatics

$528 \quad 68$, e86 (2019).

$52945 \quad$ Kim, S. ppcor: partial and semi-partial (part) correlation (version 1.1). Available 530 from https://CRAN.R-project.org/package=ppcor (2015).

53146 Wei, T. \& Simko, V. R package "corrplot": visualization of a correlation matrix 532 (version 0.84) Available from https://github.com/taiyun/corrplot (2017).

534 Acknowledgements. This work was supported by the National Science Foundation 535 (IOS-1942587 to K. Kohl) and a grant from the University of Pittsburgh Central 536 Research Development Fund. We thank A. Darracq, M. D. Dearing, T. Derting, R. 537 Martínez-Mota, and S. Secor for help with animal collections and dissections.

539 Author Contributions. K.D.K conceived the project. B.K.T designed the experiments, 540 performed the experiments, collected data, interpreted the results, and wrote the 541 manuscript with guidance from K.D.K.

543 Additional Information. Supplementary information is available for this paper. 544 Sequencing data are deposited in the NCBI SRA database under PRJNA629007. The 
bioRxiv preprint doi: https://doi.org/10.1101/2020.07.02.184382; this version posted July 2, 2020. The copyright holder for this preprint (which was not certified by peer review) is the author/funder. All rights reserved. No reuse allowed without permission.

545 authors declare no competing financial interests. Correspondence and requests for

546 materials should be addressed to B.K.T. (brian.trevelline@gmail.com). 


\section{EXTENDED DATA FIGURES AND TABLES}

a

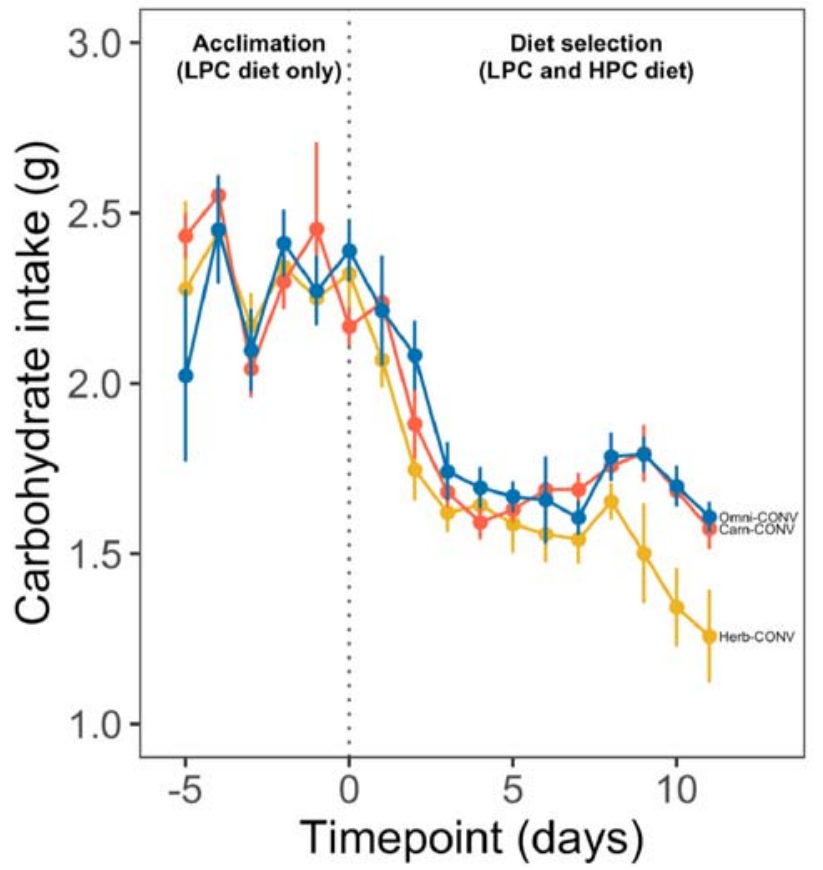

b

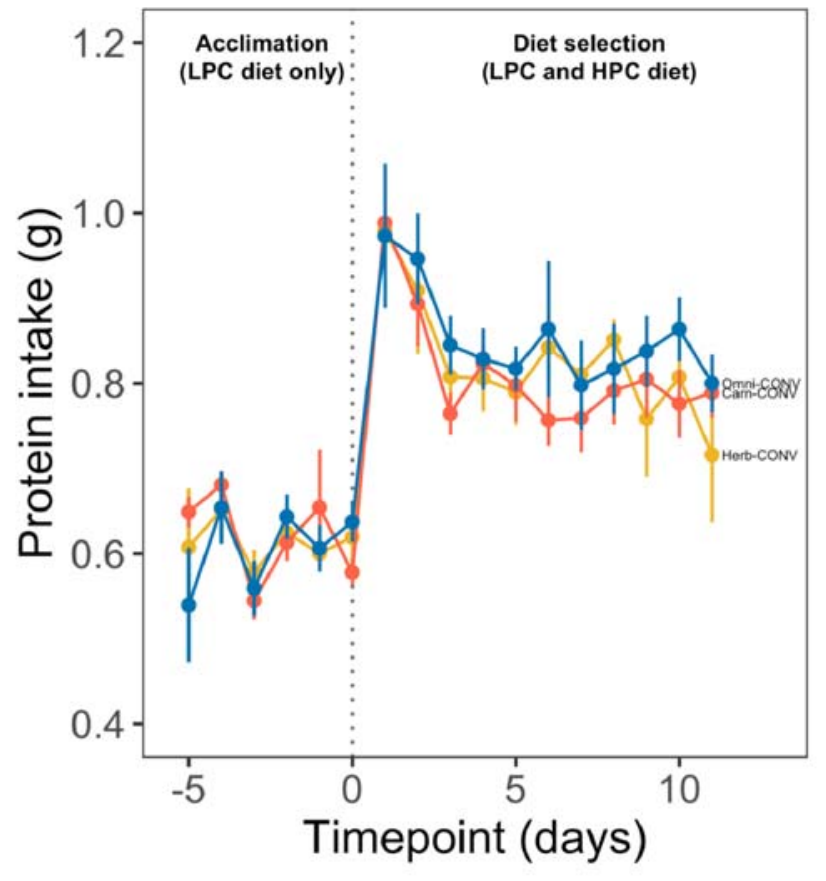

548

Extended Data Fig. 1. Daily macronutrient intake across treatment groups. a, Daily carbohydrate intake. During the acclimation period, there was no difference in either daily $(F=1.14, P=0.335)$ or cumulative carbohydrate intake $(F=0.74, P=0.483)$ across treatment groups. In contrast, there was a significant difference in daily carbohydrate intake during the diet selection experiment $(F=5.42, P=0.011)$. b, Daily protein intake. There was no difference in daily (MANOVA, $F=1.14, P=0.335$ ) or cumulative protein intake $(F=0.74, P=0.483)$ during the acclimation period, nor was there a difference in protein intake during the diet selection experiment $(F=0.71, P=$ 0.497). Line color corresponds to treatment groups: Carn-CONV (red), Omni-CONV 559 560 


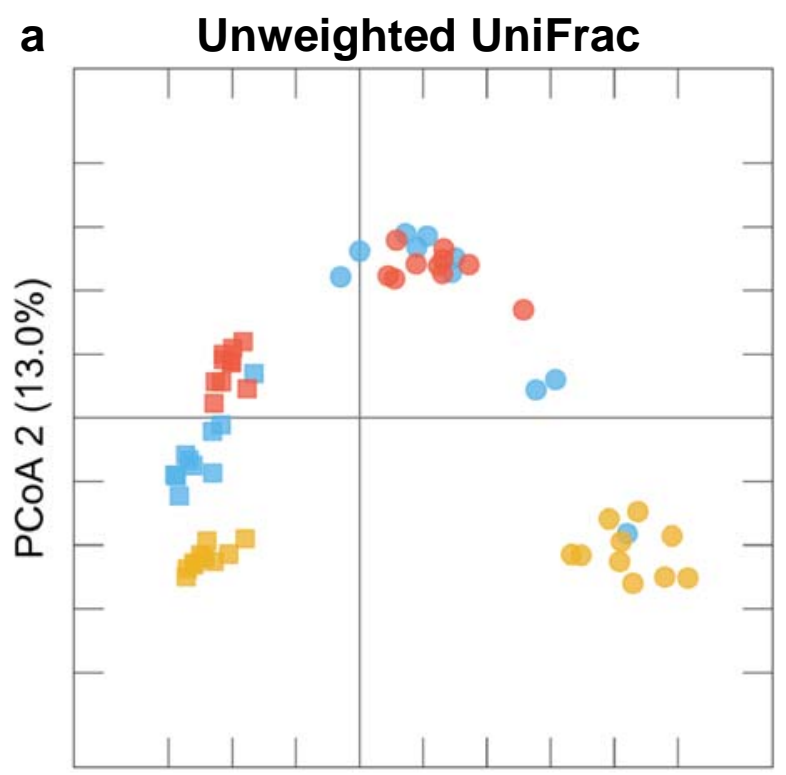

PCoA $1(24.8 \%)$

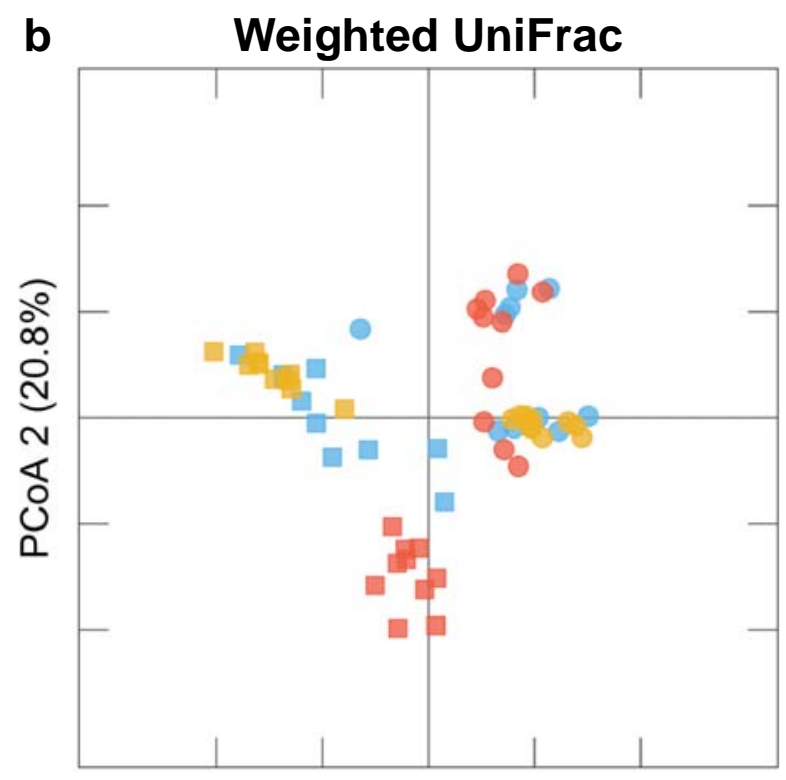

PCoA $1(31.7 \%)$

562 Extended Data Fig. 2. PCoA analysis of 16S rRNA inventories using unweighted

563 and weighted UniFrac metrics. a, Principal coordinate analysis (PCoA) of unweighted

564 UniFrac dissimilarities showing significant differences among wild donors (squares;

565 Pseudo- $\mathrm{F}=7.94, \mathrm{P}=0.001$ ) and conventionalized mice at day 0 (circles; Pseudo- $\mathrm{F}=$

$5666.33, \mathrm{P}=0.001)$. b, PCoA analysis of weighted UniFrac dissimilarities showing

567 significant differences among wild donors (squares; Pseudo- $F=14.73, P=0.001$ ) and

568 conventionalized mice at day 0 (circles; Pseudo-F $=7.35, P=0.001$ ). 
a

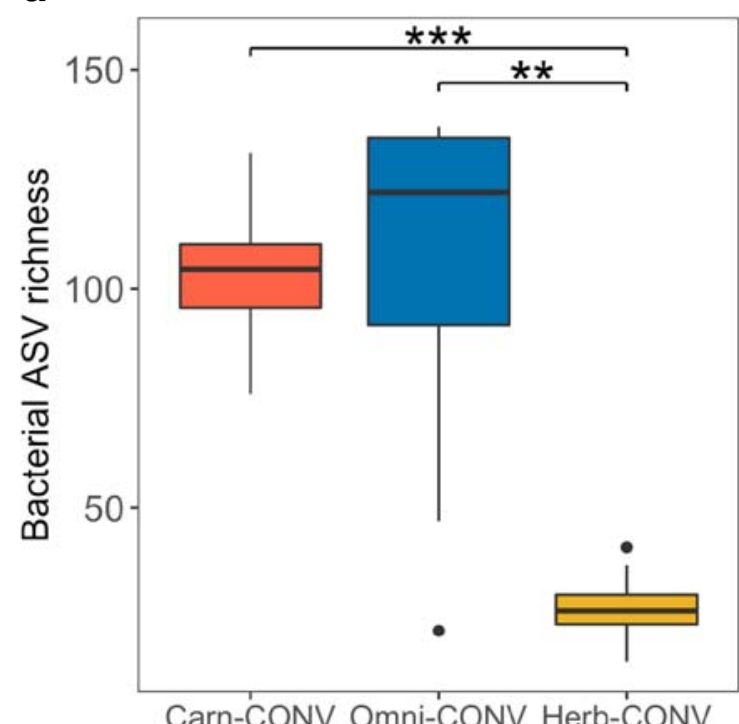

b

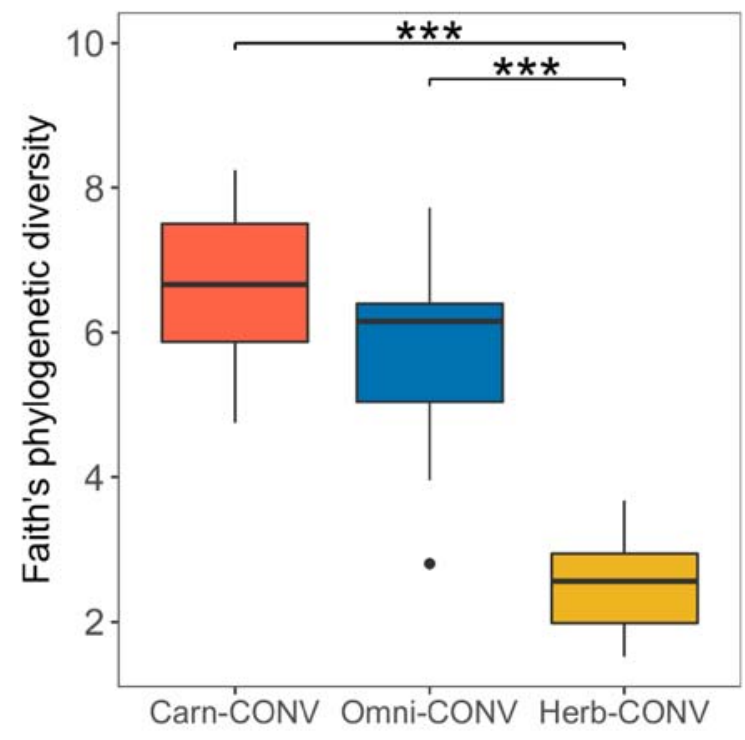

577 Extended Data Fig. 3. Differences in bacterial alpha diversity across treatment groups. a, Bacterial amplicon sequence variant (ASV) richness differed significantly 579 across treatment groups $(F=16.70, F D R$ adj. $P<0.001)$, with Herb-CONV mice 580 exhibiting lower ASV richness compared to Carn-CONV $(F=14.30, P<0.001)$ and

581 Omni-CONV mice $(F=10.09, P=0.002) . \quad b$, Faith's phylogenetic diversity differed 582 significantly across treatment groups $(F=18.50, P<0.001)$, with Herb-CONV mice 583 exhibiting lower diversity compared to Carn-CONV $(F=14.29, P<0.001)$ and Omni584 CONV mice $(F=12.09, P<0.001) .{ }^{* *}$ denotes $\mathrm{P}<0.01$ and ${ }^{* * *}$ denotes $\mathrm{P}<0.001$. 
a

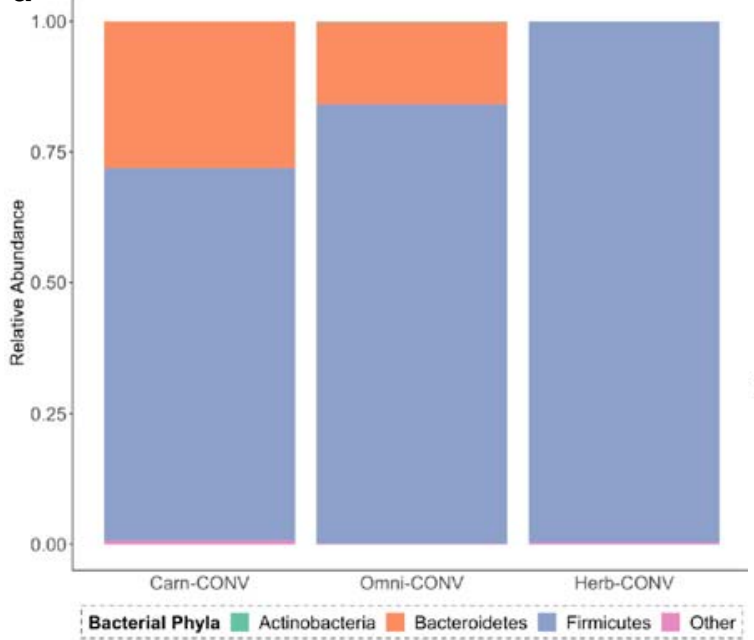

b

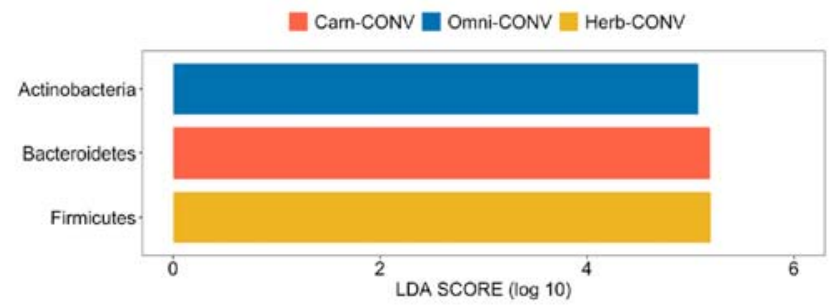

C

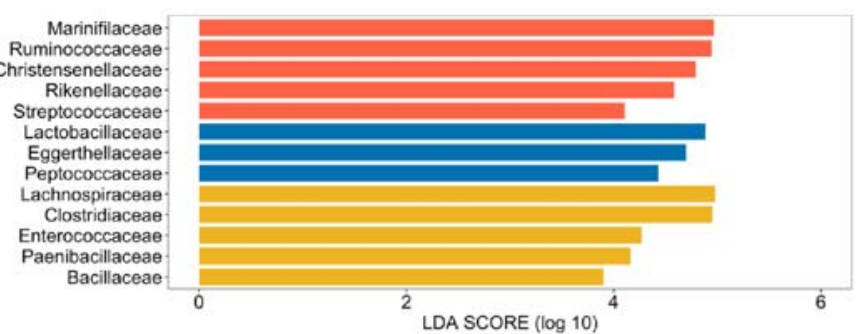

591 Extended Data Fig. 4. Differences in bacterial community composition across

592 treatment groups. a, Relative abundances of bacterial phyla across treatment groups.

593 b, Results of LEfSe analysis showing bacterial phyla that differed significantly in relative

594 abundance across treatment groups. c, Results of LEfSe analysis showing bacterial

595 families that differed significantly in relative abundance across treatment groups. 
607 Extended Data Table 1. Nutritional information of HPC and LPC diets.

\begin{tabular}{lcc}
\hline Diet composition & Low Protein:Carbohydrate diet $(\mathrm{LPC})$ & High Protein:Carbohydrate diet $(\mathrm{HPC})$ \\
\hline Protein $(\mathrm{g} / \mathrm{Kg})$ & 140.39 & 277.44 \\
$\mathrm{CHO}(\mathrm{g} / \mathrm{Kg})$ & 526.23 & 392.23 \\
Fat $(\mathrm{g} / \mathrm{Kg})$ & 50.28 & 49.75 \\
Fiber $(\mathrm{g} / \mathrm{Kg})$ & 32.90 & 40.65 \\
$\mathrm{NDF}(\mathrm{g} / \mathrm{Kg})$ & 120.09 & 119.51 \\
$\mathrm{Ca}(\mathrm{g} / \mathrm{Kg})$ & 9.99 & 10.01 \\
$\mathrm{Cl}(\mathrm{g} / \mathrm{Kg})$ & 2.77 & 2.44 \\
$\mathrm{~K}(\mathrm{~g} / \mathrm{Kg})$ & 8.23 & 7.72 \\
$\mathrm{Mg}(\mathrm{g} / \mathrm{Kg})$ & 1.67 & 1.47 \\
Na $(\mathrm{g} / \mathrm{Kg})$ & 1.36 & 1.41 \\
$\mathrm{P} \mathrm{Avail}(\mathrm{g} / \mathrm{Kg})$ & 3.48 & 3.47 \\
$\mathrm{P}(\mathrm{g} / \mathrm{Kg})$ & 5.64 & 5.11 \\
$\mathrm{~B}-12(\mathrm{mg} / \mathrm{Kg})$ & 0.03 & 0.03 \\
$\mathrm{~B}-6(\mathrm{mg} / \mathrm{Kg})$ & 21.65 & 20.47 \\
Biotin $(\mathrm{mg} / \mathrm{Kg})$ & 0.56 & 0.56 \\
Folic Acid $(\mathrm{mg} / \mathrm{Kg})$ & 2.36 & 2.35 \\
Niacin $(\mathrm{mg} / \mathrm{Kg})$ & 136.36 & 129.75 \\
Pantothenate $(\mathrm{mg} / \mathrm{Kg})$ & 68.42 & 67.71 \\
Riboflavin $(\mathrm{mg} / \mathrm{Kg})$ & 23.43 & 23.37 \\
Thiamin $(\mathrm{mg} / \mathrm{Kg})$ & 19.56 & 19.84 \\
Vit A $(\mathrm{IU} / \mathrm{Kg})$ & 19856.00 & 19888.00 \\
Vit D $(\mathrm{IU} / \mathrm{Kg})$ & 2204.50 & 2206.50 \\
Vit E $(\mathrm{IU} / \mathrm{Kg})$ & 143.92 & 138.69 \\
Vit K $(\mathrm{mg} / \mathrm{Kg})$ & 50.07 & 50.01 \\
Choline $(\mathrm{mg} / \mathrm{Kg})$ & 2074.46 & 2045.11 \\
Inositol $(\mathrm{mg} / \mathrm{Kg})$ & 1128.92 & 1372.32 \\
PABA $(\mathrm{mg} / \mathrm{Kg})$ & 110.13 & 110.13 \\
Vit C $(\mathrm{mg} / \mathrm{Kg})$ & 991.19 & 991.19 \\
\hline
\end{tabular}


617 Extended Data Table 2. Summary of microbiome similarity between

618 conventionalized recipients and their donors. In general, analysis of similarity

619 (ANOSIM) $\mathrm{R}$ values indicated that recipient microbiota closely resembled the

620 communities of their donor species. However, equal group beta dispersions

621 (PERMDISP) is a key assumption of ANOSIM, and thus in the cases of Carn-CONV

622 and Herb-CONV conventionalized recipients, the large differences in PERMDISP

623 between treatment groups may render ANOSIM results unreliable. * denotes a q-value

624 (FDR-corrected P-value) $\leq 0.05$.

\begin{tabular}{llcccc}
\hline Recipients & Donors & ANOSIM R & ANOSIM q-value & PERMDISP F & PERMDISP q-value \\
\hline Carn-CONV & O. torridus & 0.65 & $0.001^{*}$ & 20.21 & $0.003^{*}$ \\
& M. montanus & 1 & $0.001^{*}$ & 4.09 & $0.004^{*}$ \\
& P. leucopus & 0.99 & $0.001^{*}$ & 4.89 & $0.01^{*}$ \\
Omni-CONV & P. leucopus & 0.78 & $0.001^{*}$ & 0.94 & 0.365 \\
& M. montanus & 0.87 & $0.001^{*}$ & 0.77 & 0.303 \\
& O. torridus & 0.81 & $0.001^{*}$ & 9.76 & $0.003^{*}$ \\
Herb-CONV & M. montanus & 0.96 & $0.001^{*}$ & 8.64 & $0.003^{*}$ \\
& O. torridus & 0.9 & $0.001^{*}$ & 26.49 & $0.003^{*}$ \\
& P. leucopus & 0.97 & $0.001^{*}$ & 9.9 & $0.003^{*}$ \\
\hline
\end{tabular}

